\title{
Modified clustered comb pilot-aided fast time-varying channel estimation for OFDM system
}

\author{
Xin LI*, Pingzhi FAN \\ Institute of Mobile Communications, Southwest Jiaotong University, Chengdu 610031, China
}

\begin{abstract}
As high-speed railway is booming worldwide, the communication system with fast-time varying channel has drawn great attention. The comb pilot based linear minimum mean square error (LMMSE) channel estimator is proved to be an effective method for fast time-varying channel estimation. In this paper, the clustered comb pilot-aided channel estimation for orthogonal frequency-division multiplexing (OFDM) system is discussed, where the time varying channel is approximated by a basis expansion model (BEM). A modified clustered comb pilot structure is proposed and justified to improve the estimation performance compared with the clustered comb pilot proposed by Tang. Based on the complex-exponential BEM (CE-BEM) model, a suboptimal-pilot structure is proposed. In addition, optimal pilot length is analyzed and simulated with a predefined total number of pilots. The simulation results show that the modified clustered comb pilot can greatly reduce the estimation error especially with high Doppler spread. The suboptimalpilot structure with guard pilot approximation is proven to be competitive. Optimal nonzero pilot lengths for different Doppler spread are obtained by simulation with a predefined channel order and fixed pilot subcarriers.
\end{abstract}

Key words: OFDM; LMMSE; BEM; clustered comb pilot; high mobility

(C) 2012 JMT. All rights reserved.

\section{Introduction}

$\mathrm{O}$ rthogonal frequency-division multiplexing (OFDM) has been proven to be an effective technique to overcome the inter-symbol interference (ISI) caused by frequency-selective fading. OFDM is also a spectrum efficient method for the high data rate transmission, and is adopted by several wireless communication standards, such as mobile worldwide interoperability microwave systems for next-generation wireless communication systems (WiMAX), and 3GPP longterm evolution (LTE) system. Meanwhile, as high-speed railway is booming worldwide, mobility becomes an important factor. Under the high mobility scenario, the channel is no longer time-invariant during one OFDM symbol, which results in fast time-varying (TV) characteristics. Thus, reliable channel estimation for such fast fading channel plays an important role.

For such kind of channel estimation, pilot based method is particularly important. There are three kinds of pilot arrangements in OFDM system: block-type pilot, comb-type pilot, and mixed-type pilot [1-2], as shown in

Received Oct. 21, 2012; revision accepted Nov. 4, 2012

*Corresponding author. E-mail: lixin-dream@163.com (X. LI)

(C) 2012 JMT. All rights reserved

doi: 10.3969/j.issn.2095-087X.2012.04.005
Fig. 1. When the channel fades fast, the comb-type method shows the best performance followed by the mixed-type, and then block-type. The block-type pilot can not track rapid channel variation due to the large interpolation error in time domain [3]. When there are more channel paths, the comb-type method suffers from an error floor due to limited pilot clusters restricted by bandwidth. The mixed pilot is a compromise between block pilot and comb pilot. As a result of high mobility, the orthogonality of OFDM subcarriers may be destroyed, resulting in more serious inter carrier interfere-

\begin{tabular}{|c|c|c|}
\hline 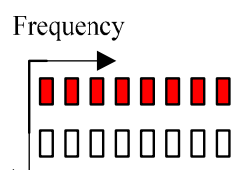 & 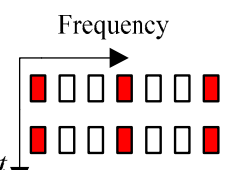 & 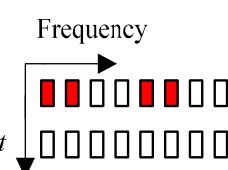 \\
\hline 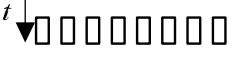 & 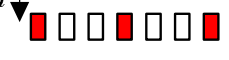 & प्र० \\
\hline 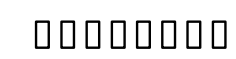 & 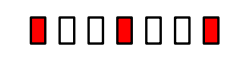 & प्रा \\
\hline 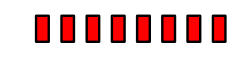 & 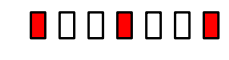 & 00 \\
\hline 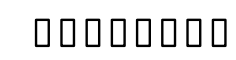 & 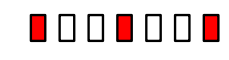 & प्र००र \\
\hline 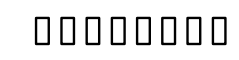 & 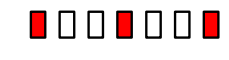 & 00 \\
\hline 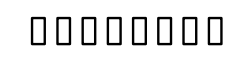 & 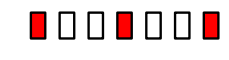 & 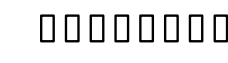 \\
\hline (a) $\mathrm{H}$ & ) & c) $\mathrm{Mix}$ \\
\hline
\end{tabular}

Fig. 1 Three kinds of pilot arrangements in OFDM system (dark square stands for pilot carrier) 
ence (ICI). To reduce the influence of ICI, a clustered comb-type pilot with zero guard was presented in Refs. [4-5], where the pilot was clustered in several groups. Within each group, one nonzero pilot was placed in the middle with some zero guard on both sides, called frequency-domain Kronecker delta (FDKD) structure. This equidistance pulse-shaped pilot arrangement was claimed to be optimal in Ref. [5]. In Ref. [6], the minimum mean square error (MMSE)-optimal pilot structure is studied, but the influences of the data subcarriers were not considered.

To our knowledge, the optimal length of guard pilot is not studied as it depends on different channel models.

Common approaches to describing the channel model are based on physical propagation parameters such as the path delay, path phases, path frequencies, etc., which are generally difficult to handle. Therefore, much existing work uses a basis expansion model (BEM), an effective model for doubly-selective channel estimation. The BEM approximates the channel taps by combining prescribed basis functions in the time domain. So far, many BEM designs have been proposed to model the channel. The discrete Karhuen-Loève BEM (DKL-BEM) [7] is optimal in terms of the mean square error (MSE). DKLBEM is in essence a reduced-rank decomposition of the Doppler spectrum, bathtub-shaped or bell-shaped. Complex-exponential BEM (CE-BEM) [8] is regarded as a special DKL-BEM with white spectrum. The frequency channel matrix for CE-BEM is strictly banded and widely used due to its algebraic simplicity. In addition, a great deal of attention is paid to polynomial BEM (PBEM) [9]. It models the channel taps using a combination of a set of polynomials with better performance in the lower Doppler spread than in the higher Doppler spread.

For the channel estimator, linear minimum mean square error (LMMSE) has been taken into consideration, while the BEM coefficient vector is independent of transmitted data and noise. Tang et al. proposed a suboptimal LMMSE estimator [10], which uses the assumed channel statistic properties but not the real-time calculation, achieving complexity reduction with sacrifices of accuracy.

In this paper, on the basis of the LMMSE estimator in Ref. [10] and the CE-BEM channel model, clustered and modified clustered comb pilot structures are introduced, and channel estimation performances of the two different structures are compared. Besides, a suboptimal-pilot structure with guard pilot approximation is proposed. The guard pilot length and nonzero pilot length are also analyzed. Various simulation results and discussions especially for the optimal choice of nonzero pilot are provided.

\section{System model}

\subsection{CE-BEM channel model}

Let us define $h(n, l)$ as the $l$ th channel tap at the $n$th time instant, $n=0,1, \cdots, N-1 . \quad N$ represents the sampling number for each path, and $h(n, l)=0$ for $l<0$ or $l>L-1$, where $L$ is the number of channel paths. By using a BEM model, $h(n, l)$ can be represented as follows [8]:

$$
h(n, l)=\sum_{q=0}^{Q} c_{q}(l) b_{q}(n)
$$

where $c_{q}(l)$ is the $(q+1)$ th BEM coefficients of the $l$ th channel tap during one OFDM symbol; $\boldsymbol{b}_{q}$ is the basis function with size $N \times 1 ; Q=2\left\lceil f_{\mathrm{d}} N T_{\mathrm{s}}\right\rceil, \quad f_{\mathrm{d}}$ is the maximum Doppler shift and calculated as $f_{\mathrm{d}}=f_{\mathrm{c}} v / c$, $f_{\mathrm{c}}$ is central carrier frequency, and $T_{\mathrm{s}}$ is the sampling period.

The basis function matrix can be written as

$$
\boldsymbol{B}=\left[\begin{array}{llll}
\boldsymbol{b}_{0} & \boldsymbol{b}_{1} & \cdots & \boldsymbol{b}_{q}
\end{array}\right], \quad \boldsymbol{B}=\left[B_{p, q}\right]_{N \times(Q+1)} .
$$

For CE-BEM, complex basis function is used as the basis:

$$
B_{p, q}=\exp \left(\mathrm{j} \frac{2 \pi}{N}\left(q-\frac{Q}{2}\right)\right) .
$$

\subsection{OFDM system model}

We consider an OFDM system with $N$ subcarriers. To avoid inter symbol interference (ISI), cyclic prefix of $L_{\mathrm{cp}}$ is added. $L_{\mathrm{cp}}$ is chosen to be equal to or greater than the channel's maximum delay. Throughout the paper, only one OFDM symbol is considered. The time-domain transmitted signal $\boldsymbol{x}$ can be written as

$$
\begin{aligned}
x(n) & =\frac{1}{\sqrt{N}} \sum_{k=0}^{N-1} X(k) \exp \left(\mathrm{j} \frac{2 \pi}{N} k n\right), \\
n & =-L_{\mathrm{cp}},-L_{\mathrm{cp}}+1, \cdots, N-1 .
\end{aligned}
$$

After removing the cyclic prefix, the received signal can be represented as

$$
y(n)=\sum_{l=0}^{L-1} h(n, l) x(n-l)+w(n), \quad n=0,1, \cdots, N-1,
$$

where $w(n)$ is Gaussian noise in time domain with zero mean and variance $N_{0}$. 
Then a discrete Fourier transform (DFT) is performed and the received signal in frequency domain $\boldsymbol{Y}$ is obtained:

$$
Y(n)=\boldsymbol{F} y(n)=\boldsymbol{F} h(n) \boldsymbol{F}^{\mathrm{H}} X(n)+W(n),
$$

where $\boldsymbol{F}$ is an $N$ point unitary discrete Fourier transform (DFT) matrix:

$$
\boldsymbol{F}=\left[F_{p, q}\right]_{N \times N}, \quad F_{p, q}=\frac{1}{\sqrt{N}} \exp \left(-\mathrm{j} \frac{2 \pi}{N} p q\right) ;
$$

the transmitted signal in frequency domain $\boldsymbol{X}=\boldsymbol{F} \boldsymbol{x} ; \boldsymbol{h}$ is time domain channel matrix $\boldsymbol{h}=\left[h_{p, q}\right]_{N \times N}$ with $h_{p, q}=h(p, \bmod (p-q, N)) ;$ and $W(n)$ denotes channel noise in the frequency domain.

The frequency domain channel matrix can be written as $\boldsymbol{H}=\boldsymbol{F} \boldsymbol{h} \boldsymbol{F}^{\mathrm{H}}$. For time-invariant channel, $\boldsymbol{H}$ is diagonal. When the channel is time-varying, however, $\boldsymbol{H}$ is no longer diagonal with energy spread out to the whole frequency band, but primarily neighboring subcarriers. Thus $\boldsymbol{H}$ can be approximated as banded which coincides with the property of CE-BEM.

Combined with Eq. (1), we can rewrite the time domain channel matrix $\boldsymbol{h}$ as

$$
\boldsymbol{h}=\sum_{q=0}^{Q} \operatorname{diag}\left(\boldsymbol{b}_{q}\right) \boldsymbol{C}_{q},
$$

where $\boldsymbol{C}_{q}$ is a circulate matrix formed by $\left[\boldsymbol{c}_{q}{ }^{\mathrm{T}} 0 \cdots 0\right]^{\mathrm{T}}$

$$
\boldsymbol{C}_{q}=\left[\begin{array}{ccccccc}
c_{q, 0} & 0 & \cdots & 0 & c_{q, L-1} & \cdots & c_{q, 1} \\
c_{q, 1} & c_{q, 0} & 0 & \cdots & 0 & 0 & c_{q, 2} \\
\vdots & \ddots & \ddots & \ddots & \ddots & \ddots & \vdots \\
c_{q, L-1} & \ddots & \ddots & \ddots & \ddots & \ddots & 0 \\
0 & \ddots & \ddots & \ddots & \ddots & \ddots & 0 \\
\vdots & \ddots & \ddots & \ddots & \ddots & \ddots & \vdots \\
0 & \cdots & \cdots & c_{q, L-1} & \cdots & \cdots & c_{q, 0}
\end{array}\right] .
$$

Substituting (6) into (5), one can obtain

$$
\begin{gathered}
\boldsymbol{Y}=\sum_{q=0}^{Q} \boldsymbol{F} \operatorname{diag}\left(\boldsymbol{b}_{q}\right) \boldsymbol{C}_{q} \boldsymbol{F}^{\mathrm{H}} \boldsymbol{X}+\boldsymbol{W}= \\
\sum_{q=0}^{Q} \boldsymbol{F} \operatorname{diag}\left(\boldsymbol{b}_{q}\right) \boldsymbol{F}^{\mathrm{H}} \operatorname{diag}\left(\boldsymbol{F}_{L} \boldsymbol{c}_{q}\right) \boldsymbol{X}+\boldsymbol{W}= \\
\sum_{q=0}^{Q} \boldsymbol{F} \operatorname{diag}\left(\boldsymbol{b}_{q}\right) \boldsymbol{F}^{\mathrm{H}} \operatorname{diag}(\boldsymbol{X}) \boldsymbol{F}_{L} \boldsymbol{c}_{q}+\boldsymbol{W},
\end{gathered}
$$

where $\boldsymbol{F}_{L}$ stands for the first $L$ columns of matrix.

\subsection{LMMSE estimator}

In this paper, the LMMSE estimator [10] is adopted under two assumptions: (1) the BEM coefficients matrix $\boldsymbol{c}=\left[\boldsymbol{c}_{0}{ }^{\mathrm{T}} \boldsymbol{c}_{1}{ }^{\mathrm{T}} \cdots \boldsymbol{c}_{Q}{ }^{\mathrm{T}}\right]^{\mathrm{T}}$ is independent of data and noise, and the data is also independent of noise; (2) data is zero-mean, white, with variance and uncorrelated with the noise.

In the following discussion, we define $\boldsymbol{M}^{(\mathrm{p})}$ to be a sub-matrix of $\boldsymbol{M}$ which collects the elements corresponding to the pilots, while $\boldsymbol{M}^{(\mathrm{d})}$ collects the elements corresponding to the data in $\boldsymbol{M}$. The received signal corresponding to the nonzero pilot can be expressed as

$$
\boldsymbol{Y}^{(\mathrm{p})}=\boldsymbol{A}^{(\mathrm{p})} \boldsymbol{\Delta}^{(\mathrm{p})} \boldsymbol{c}+\boldsymbol{d}+\boldsymbol{W}=\boldsymbol{D} \boldsymbol{c}+\boldsymbol{d}+\boldsymbol{W},
$$

where the first term $\boldsymbol{D} \boldsymbol{c}$ represents signal from pilot, the second term $\boldsymbol{d}$ is the interferences from data, $\boldsymbol{W}$ is Gaussian noise, $\boldsymbol{D}=\boldsymbol{A}^{(\mathrm{p})} \boldsymbol{\Delta}^{(\mathrm{p})}, \boldsymbol{d}=\boldsymbol{A}^{(\mathrm{d})} \boldsymbol{\Delta}^{(\mathrm{d})}$,

$$
\begin{aligned}
& \boldsymbol{\Delta}^{(\mathrm{p})}=\boldsymbol{I}_{Q+1} \otimes\left(\operatorname{diag}\left(\boldsymbol{X}^{(\mathrm{p})}\right) \boldsymbol{F}_{L}^{(\mathrm{p})}\right), \\
& \boldsymbol{A}^{(\mathrm{p})}=\left[\boldsymbol{A}_{1}^{(\mathrm{p}) \mathrm{T}}, \cdots, \boldsymbol{A}_{M}^{(\mathrm{p}) \mathrm{T}}\right]^{\mathrm{T}}=\left[\begin{array}{ccc}
\boldsymbol{A}_{0,1}^{(\mathrm{p})} & \cdots & \boldsymbol{A}_{Q, 1}^{(\mathrm{p})} \\
\vdots & \ddots & \vdots \\
\boldsymbol{A}_{0, M}^{(\mathrm{p})} & \cdots & \boldsymbol{A}_{Q, M}^{(\mathrm{p})}
\end{array}\right],
\end{aligned}
$$

$\boldsymbol{A}_{q, m}^{(\mathrm{p})}$ is sub-matrix of $\boldsymbol{A}_{q}, \quad \boldsymbol{A}_{q}=\boldsymbol{F} \operatorname{diag}\left(\boldsymbol{b}_{q}\right) \boldsymbol{F}^{\mathrm{H}}$, which collects rows corresponding to nonzero pilots in the $m$ th cluster and columns corresponding to all pilots. $\boldsymbol{A}_{q, m}^{(\mathrm{d})}$ collects rows corresponding to nonzero pilots in the $m$ th cluster and columns corresponding to data. For more details, please refer to Ref. [10].

The LMMSE estimator treats $c$ as a stochastic variable. We seek a linear filter $\boldsymbol{V}$ such that the MSE between the estimated BEM coefficients and the true BEM coefficients is minimal:

$$
\begin{aligned}
\boldsymbol{V} & =\arg \min \operatorname{trace}\left\{E\left\{\left(\boldsymbol{V} \boldsymbol{Y}^{(\mathrm{p})}-\boldsymbol{c}\right)\left(\boldsymbol{V} \boldsymbol{Y}^{(\mathrm{p})}-\boldsymbol{c}\right)^{\mathrm{H}}\right\}\right\}= \\
& \boldsymbol{R}_{c} \boldsymbol{D}^{\mathrm{H}}\left(\boldsymbol{D} \boldsymbol{R}_{c} \boldsymbol{D}^{\mathrm{H}}+\boldsymbol{R}_{d}+\boldsymbol{R}_{W}^{(\mathrm{p})}\right)^{-1}= \\
& \boldsymbol{R}_{c} \boldsymbol{D}^{\mathrm{H}}\left(\boldsymbol{D} \boldsymbol{R}_{c} \boldsymbol{D}^{\mathrm{H}}+\boldsymbol{R}_{I}\right)^{-1},
\end{aligned}
$$

where $\boldsymbol{R}_{c}, \boldsymbol{R}_{d}$, and $\boldsymbol{R}_{W}^{(\mathrm{p})}$ represent the covariance matrix of $\boldsymbol{c}, \boldsymbol{d}$, and $\boldsymbol{W}^{(\mathrm{p})}$ respectively. $\boldsymbol{d}$ carries the channel information $c$, so extra task is needed for LMMSE estimator in [10] compared with LMMSE estimator in [11]. In this paper, we treat interference as stochastic variable, and focus on the statistical property of $\boldsymbol{c}$ and $\boldsymbol{d}$.

First we start with the coefficient of the $l$ th path BEM, $c_{l}$. The autocorrelation matrix can be calculated as

$$
\boldsymbol{R}_{\boldsymbol{c}_{l}}=E\left\{\boldsymbol{c}_{l} \boldsymbol{c}_{l}^{\mathrm{H}}\right\}=\boldsymbol{B}^{-1} E\left\{h(n, l) h^{*}(m, l)\right\} \boldsymbol{B}^{-1 \mathrm{H}},
$$

where $E\left\{h(n, l) h^{*}(m, l)\right\}$ is determined by the Doppler spectrum. In this paper, Jakes model [12] is employed:

$$
E\left\{h(n, l) h^{*}(m, l)\right\}=J_{0}\left(2 \pi f_{\mathrm{d}}(n-m) T_{\mathrm{s}}\right) .
$$


Then $\boldsymbol{R}_{c}$ can be written as

$$
\boldsymbol{R}_{c}=\boldsymbol{R}_{c_{l}} \otimes \boldsymbol{R}_{L},
$$

where $\boldsymbol{R}_{L}$ describes the correlation due to channel's frequency selectivity. We take the notation in [13],

$$
\boldsymbol{R}_{L}=\operatorname{diag}\left[\begin{array}{llll}
\sigma_{0}^{2} & \sigma_{1}^{2} & \cdots & \sigma_{L-1}^{2}
\end{array}\right],
$$

with $\sigma_{l}^{2}=\mathrm{e}^{-l / 10}$.

With the help of resolved $\boldsymbol{R}_{L}$, we can derive the covariance matrix of interference term $\boldsymbol{d}$ :

$$
\begin{aligned}
& \boldsymbol{R}_{\boldsymbol{d}}=E\left\{\boldsymbol{d} \boldsymbol{d}^{\mathrm{H}}\right\}=E\left\{\boldsymbol{A}^{(\mathrm{d})} \boldsymbol{\Delta}^{(\mathrm{d})} \boldsymbol{c} \boldsymbol{c}^{\mathrm{H}} \boldsymbol{\Delta}^{(\mathrm{d}) \mathrm{H}} \boldsymbol{A}^{(\mathrm{d}) \mathrm{H}}\right\}= \\
& \boldsymbol{A}^{(\mathrm{d})} E\left\{\Delta^{(\mathrm{d})} \boldsymbol{c} \boldsymbol{c}^{\mathrm{H}} \boldsymbol{\Delta}^{(\mathrm{d}) \mathrm{H}}\right\} \boldsymbol{A}^{(\mathrm{d}) \mathrm{H}}= \\
& \boldsymbol{A}^{(\mathrm{d})} \boldsymbol{R}_{x} \boldsymbol{A}^{(\mathrm{d}) \mathrm{H}}
\end{aligned}
$$

where

$$
\begin{aligned}
& \boldsymbol{R}_{x}=E\left\{\boldsymbol{\Delta}^{(\mathrm{d})} \boldsymbol{c} \boldsymbol{c}^{\mathrm{H}} \boldsymbol{\Delta}^{(\mathrm{d}) \mathrm{H}}\right\}= \\
& \quad E\left\{\left(\boldsymbol{I}_{Q+1} \otimes \operatorname{diag}\left(\boldsymbol{X}^{(\mathrm{d})}\right)\right) \chi\left(\boldsymbol{I}_{Q+1} \otimes \operatorname{diag}\left(\boldsymbol{X}^{(\mathrm{d})}\right)\right)^{\mathrm{H}}\right\},
\end{aligned}
$$

with $\chi=\left(\boldsymbol{I}_{Q+1} \otimes \boldsymbol{F}_{L}^{(\mathrm{d})}\right) \boldsymbol{R}_{\boldsymbol{c}}\left(\boldsymbol{I}_{Q+1} \otimes \boldsymbol{F}_{L}^{(\mathrm{d})}\right)^{\mathrm{H}}$.

As a result,

$$
\left[R_{X}\right]_{m \times n}= \begin{cases}\sigma_{X}^{2}[\chi]_{m \times n} & \text { if } \bmod \left(m-n, N-M L_{\mathrm{p}}\right)=0, \\ 0 & \text { others. }\end{cases}
$$

Then substituting $\boldsymbol{R}_{c}, \boldsymbol{R}_{d}$, and $\boldsymbol{R}_{W}^{(\mathrm{p})}$ into Eq. (10), $\boldsymbol{c}$ can be estimated as $\widehat{\boldsymbol{c}}=\boldsymbol{V} \boldsymbol{Y}^{(\mathrm{p})}$.

\section{Clustered comb-pilot and modified clus- tered comb-pilot}

The effective method for TV channel estimation is to insert comb pilots at transmitter. Let's consider a frequency domain clustered comb pilot. That is, $M$ pilot clusters are inserted to each OFDM symbol with the length $L_{\mathrm{p}}+2 L_{\mathrm{g}}$ for each cluster, where $L_{\mathrm{p}}$ is the length of nonzero pilot in each cluster, while $L_{\mathrm{g}}$ is the length of zero guard at each side.

As discussed in Refs. [5] and [10], in each pilot cluster, only one nonzero pilot is inserted and several zero guards added on each side to separate the data, known as FDKD. While in a high-mobility scenarios, the fast TV channel matrix in frequency domain is non-diagonal, which leads to ICI. The guard pilot could decrease ICI impact. This kind of clustered comb-pilot is thought to be optimal in Ref. [5]. But for the channel estimation, only nonzero pilots are considered in the calculation of BEM coefficients. Too many guard pilot subcarriers is a waste of spectrum, as ICI mainly comes from the

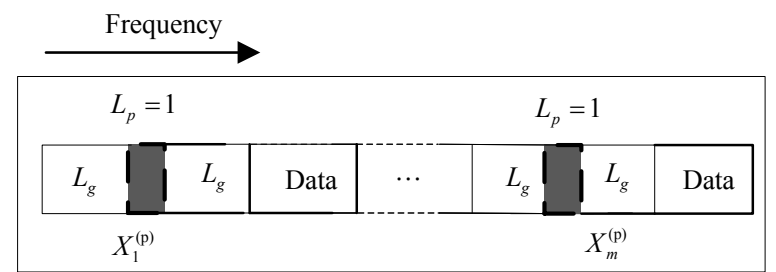

(a) Clustered comb pilot [10]

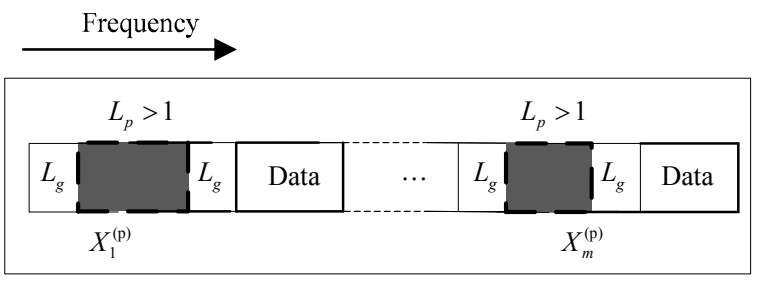

(b) Modified clustered comb pilot

Fig. 2 Clustered and modified pilots

neighboring subcarriers [14-15]. Based on the clustered comb pilot, the structure of each cluster is modified by inserting more nonzero pilot and deleting some zero guard pilot in each cluster, as shown in Fig. 2. With the same pilot number, the modified structure has more nonzero pilots, which means more received pilot symbols.

Nonzero pilot of each pilot cluster is denoted as

$$
\boldsymbol{X}_{m}^{(\mathrm{p})}=\left(X_{P_{m}}, X_{P_{m}+1}, \cdots, X_{P_{m}+L_{\mathrm{p}}-1}\right), m=1,2, \cdots, M,
$$

where $P_{m}$ stands for the position of the first nonzero pilot of the $m$ th cluster. The clustered comb pilot in [10] takes $L_{\mathrm{g}}=Q$. According to the former analysis, in the CE-BEM model based estimation, $\boldsymbol{A}_{q}$ is strictly banded and is an identity matrix but shifted by $q-Q / 2$ columns, indicating that the ICI comes from the neighboring $Q$ subcarriers. Thus, for LMMSE estimator based on the CE-BEM, the theoretical value should be $Q / 2$, since only the neighboring $Q$ subcarriers produce ICI.

In Ref. [12], while $f_{n d}=0.9$, nearly $90 \%$ of the ICI comes from the first neighboring subcarrier. Table 1 shows the interference of the $k$ th subcarrier from the $(k-1)$ th subcarrier and the $(k+1)$ th subcarrier under different normalized Doppler spread.

Table 1 Energy percentage of ICI from the first neighboring subcarrier

\begin{tabular}{cc} 
Normalized Doppler spread & Energy percentage of ICI (\%) \\
\hline 0.1 & 62 \\
0.3 & 65 \\
0.5 & 72 \\
0.9 & 89
\end{tabular}


In Table 1, the normalized Doppler spread is defined as $f_{\text {nd }}=f_{\mathrm{d}} T_{\mathrm{s}} N$, and $T_{\mathrm{s}} N$ is the duration of one OFDM symbol.

Considering the ICI mainly from the first neighboring subcarrier, to simplify the comb-type pilot, $L_{\mathrm{g}}$ can be set to 1 at the sacrifice of a bit of accuracy, but with an increased spectrum efficiency.

For the modified clustered comb pilot, multiple nonzero pilots are inserted into each cluster. To find the optimal nonzero pilot length $L_{\mathrm{p}}$, the number of pilots should not exceed $N$, and the channel estimator requires $\boldsymbol{D}$ to have full column rank [16]. Thus a necessary condition is that $\boldsymbol{D}$ should be tall or at least square, or the number of equations should not be less than the unknowns to be estimated, that is,

$$
\begin{aligned}
& M\left(L_{p}+2 L_{g}\right)<N, \\
& M\left(L_{p}+2 L_{g}\right) \geq L(Q+1) .
\end{aligned}
$$

In addition, based on the channel identifibility, there exists

$$
L \leq M \leq \frac{N}{Q+1} .
$$

To minimize the estimation MSE, for LMMSE estimator,

$$
\begin{aligned}
& M S E=E\left\{\left\|\left(\boldsymbol{B} \otimes \boldsymbol{I}_{L}\right) \hat{\boldsymbol{c}}-\left(\boldsymbol{B} \otimes \boldsymbol{I}_{L}\right) \boldsymbol{c}\right\|^{2}\right\}= \\
& \operatorname{trace}\left\{\left(\boldsymbol{D}^{H} \boldsymbol{R}_{I}^{-1} \boldsymbol{D}+\boldsymbol{R}_{\boldsymbol{c}}^{-1}\right)^{-1}\right\} .
\end{aligned}
$$

For an optimized comb pilot, $M$ and $L_{\mathrm{p}}$ should be chosen to minimize the MSE. It is difficult to find out a closed form of optimal nonzero pilot length $L_{\mathrm{p}}$ for each cluster or the number of clusters $M$. But fortunately with inequalities (15), (16) and (17), exhaustive search can be avoided.

From the above discussion, it is noted that pilot cluster length and the number of pilot clusters are all related to Doppler spread. In this paper, the number of basis functions, $Q+1$, and channel delay, $L$, are fixed and invariant with Doppler spread, producing the same bounds on the number of pilot clusters. BEM order $Q$ varies with different Doppler spread, thus the bounds on three comb pilot parameters: nonzero pilot length, zero guard pilot length, and the number of clusters, varies with Doppler spread. As a result, the optimal pilot length for each cluster will vary with Doppler spread. Besides, if clustered comb pilot is inserted adaptively depending on different Doppler spreads, the estimation performance can be improved.

\section{Simulation results and analysis}

Table 2 shows the simulation parameters used in this section, and the power of data and pilot is set to 1 .

In the simulation, the total number of pilot subcarriers is set to be the same for both comb pilot structures. Fig. 3 shows the estimation performance. With a high Doppler spread, the performance of clustered comb pilot structure [10] seems to be invariant with SNR, due to the dominant constraint of ICI. It is clear that in high Doppler spread scenario, such as $f_{\text {nd }}=0.8$, the modified clustered comb pilot has much lower MSE compared with the clustered pilot structure, with MSE decreased by $78 \%$ when SNR is $35 \mathrm{~dB}$ and MSE decreased by $63 \%$ when SNR is $5 \mathrm{~dB}$. While under lower Doppler frequency, it is a bit complicated.

When SNR is less than $13 \mathrm{~dB}$, the structure in Ref. [10] seems better; when SNR is greater than $13 \mathrm{~dB}$, however, it's opposite. The reason is that for low Doppler frequency channel, ICI is not dominant, and noise is the main constraint to the performance. The comb pilots in Ref. [10] are placed more dispersedly in frequency domain, and thus has better performance in low SNR.

Table 2 Simulation parameters

\begin{tabular}{ccc}
\hline Parameter & Variable & Value \\
\hline Carrier frequency & $F_{\mathrm{c}}(\mathrm{GHz})$ & 2 \\
FFT size & $N$ & 256 \\
Length of CP & $L_{\mathrm{cp}}$ & 30 \\
Channel order & $L$ & 6 \\
Sampling rate & $T_{\mathrm{s}}(\mathrm{s})$ & $10^{-6}$ \\
BEM order & $Q$ & 4 \\
Pilot carrier number & $N_{\mathrm{p}}$ & 64 \\
\hline
\end{tabular}

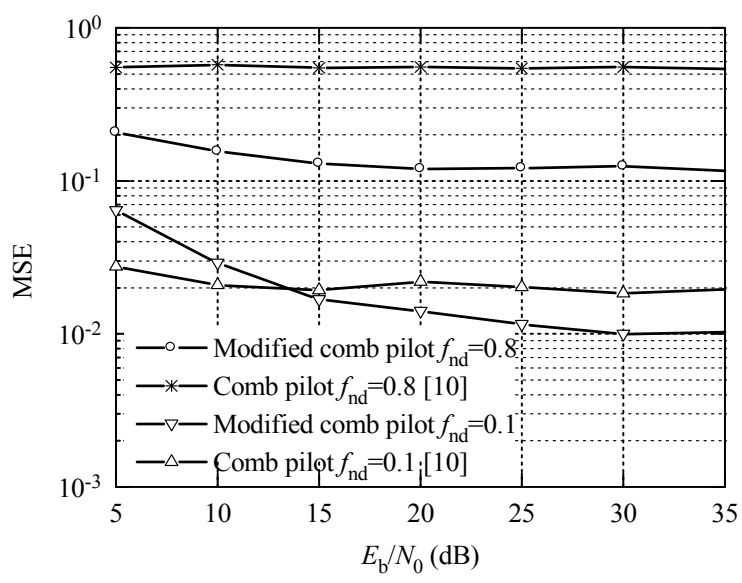

Fig. 3 Comparison between modified structure and that in Ref. [10] 
For high SNR, the modified structure decreases MSE by $46 \%$. Thus, the clustered comb pilot and modified clustered comb pilot can be adopted adaptively depending on SNR, when the Doppler spread is low.

As discussed in Section 3, for CE-BEM based channel estimation, $L_{\mathrm{g}}$ is set to be $Q / 2=2$ theoretically [17]. As shown in Fig. 4, the performance under approximated $L_{\mathrm{g}}$ is comparable with that of theoretical $L_{\mathrm{g}}$. When the normalized Doppler spread equals 0.1, the performance degradation with guard pilot approximation is slightly greater than the degradation under normalized Doppler spread 0.8. This result coincides with the ICI distribution in the first neighboring subcarrier in Table 1.

To obtain the optimal nonzero pilot length for the modified clustered comb pilot, simulation is made, and the results are shown in Fig. 5. We adopt the approximated guard pilot, that is, $L_{\mathrm{g}}=1$, and for different $L_{\mathrm{p}}$, we choose different cluster number $M$ to keep the total

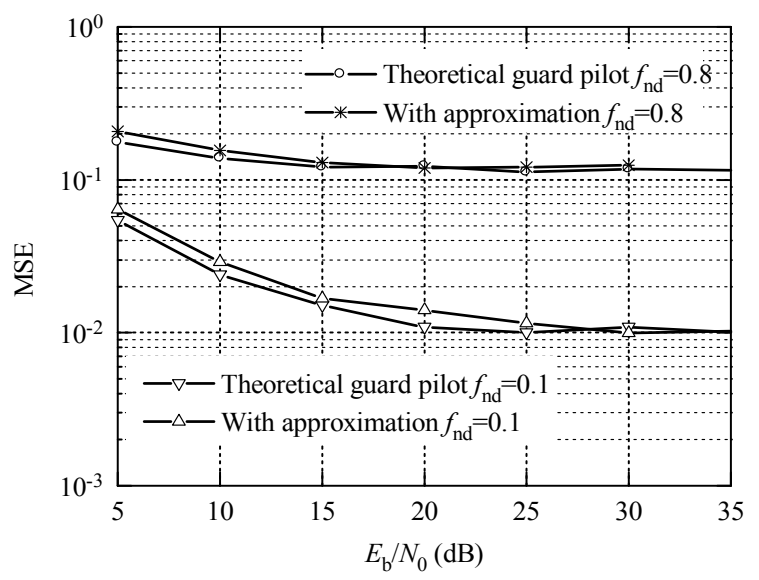

Fig. 4 Comparison of MSE performance between guard pilot approximation and theoretical one

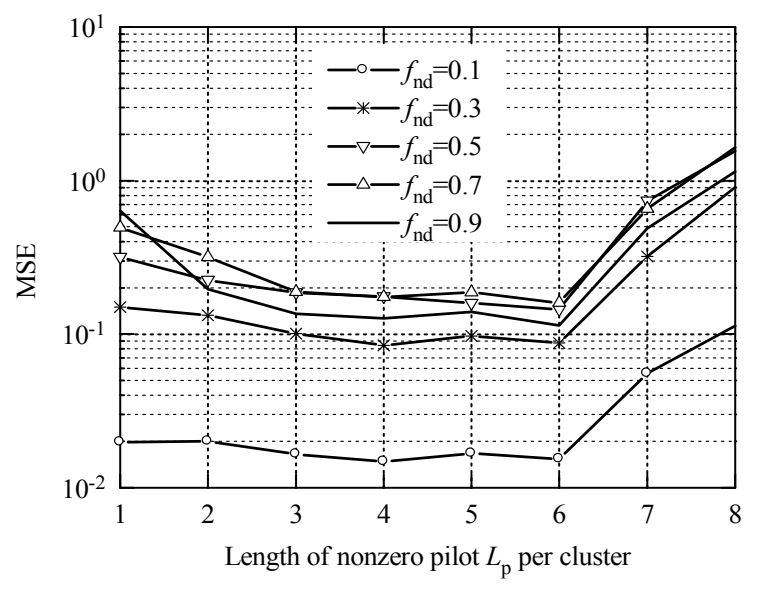

Fig. 5 MSE performance vs. nonzero pilot length $L_{\mathrm{p}}$ for different Doppler spreads (SNR is $20 \mathrm{~dB}$ ) number of pilots the same or very close, to guarantee the same spectrum efficiency. According to the previous analysis, $M \geq L$. We take $L=6$ in this paper, which is the same as that in [10]. Thus, 8 is the maximum of $L_{\mathrm{p}}$. The simulation results show that the optimal value of $L_{\mathrm{p}}$ locates in the interval of 3-6, which is in the middle of the possible range of $L_{\mathrm{p}}$. When $f_{\text {nd }}=0.1$ and $f_{\text {nd }}=0.3$, the optimal nonzero pilot length is 4 , as shown in Fig. 5. For the other three different normalized Doppler spreads, the best MSE performance is obtained if nonzero pilot length $L_{\mathrm{p}}$ is 6 .

\section{Conclusions}

In this paper, a modified clustered comb-type pilot is proposed and is compared with the clustered comb-type pilot in [10]. The simulation results show that the modified pilot help MSE of estimation decrease, especially in high Doppler spread scenarios. When Doppler spread is low, the performance is improved when SNR is bigger than $13 \mathrm{~dB}$. Besides, the energy percentage of ICI from different subcarriers is discussed. For the CE-BEM model based LMMSE estimator, an approximation of guard pilot is made to simplify the pilot. Then under the constraint of cluster number and nonzero pilot length constraint, the optimal clustered comb pilot is studied by simulation with a predefined total pilot subcarrier number. For the given example, when $L=6$, it is concluded that the optimal nonzero pilot length $L_{\mathrm{p}}$ locates in the interval of $3-6$, which is in the middle of the possible range of $L_{\mathrm{p}}$. The optimal nonzero pilot length varies with Doppler spread. The clustered comb pilot structure can be chosen based on Doppler spread or SNR. With an appropriate pilot cluster length, the estimation performance under high Doppler spread may be better than that in low Doppler spread scenario. The clustered comb pilot and modified clustered comb pilot can also be adopted adaptively depending on SNR when the Doppler spread is low.

\section{References}

[1] S. Coleri, M. Ergen, A. Puri, et al., Channel estimation techniques based on pilot arrangement in OFDM systems, Broadcasting, IEEE Transactions on, 2002, 48(3): 223-229.

[2] P. Schniter, On doubly dispersive channel estimation for pilot-aided pulse-shaped multi-carrier modulation, In: Proceedings of 2006 40th Annual Conference on Information Sciences and Systems, Princeton, NJ, Mar. 22-24, 2006: 1296-1301. 
[3] Z.J. Tang, G. Leus, Pilot schemes for time-varying channel estimation in OFDM systems, In: Signal Processing Advances in Wireless Communications, 2007, Helsinki, June 17-20, 2007: 1-5.

[4] L. Martinez, P. Ochandiano, I. Sobron, et al., Novel pilot structures for BEM channel estimation and ICI compensation in high-mobility DVB, In: Proc. of 2011 IEEE International Symposium on Broadband Multimedia Systems and Broadcasting (BMSB), Nuremberg, Jun. 8-10, 2011: 1-4.

[5] X.L. Ma, G.B. Giannakis, S. Ohno, Optimal training for block transmissions over doubly-selective fading channels, Signal Processing, 2003, 51(5): 1351-1366.

[6] K.M.Z. Islam, T.Y. Al-Naffouri, N. Al-Dhahir, On optimum pilot design for comb-type OFDM transmission over doubly-selective channels, Communications, 2011, 59(4): 930-935.

[7] K.A.D. Teo, S. Ohno, Optimal MMSE finite parameter model for doubly-selective channels, In: Proc. of Global Telecommunications Conference, 2005 (GLOBECOM'05), IEEE, St. Louis, MO, Dec. 2, 2005: 3503-3507.

[8] H.A. Cirpan, M.K. Tsatsanis, Maximum likelihood blind channel estimation in the presence of Doppler shifts, Signal Processing, 1999, 47(6): 1559-1569.

[9] S. Tomasin, A. Gorokhov, H.B. Yang, et al., Iterative interference cancellation and channel estimation for mobile
OFDM, Wireless Communications, 2005, 4(1): 238-245.

[10] Z.J. Tang, R.C. Cannizzaro, G. Leus, et al., Pilot-assisted time-varying channel estimation for OFDM systems, Signal Processing, 2007, 55(5): 2226-2238.

[11] S.M. Kay, Fundamentals of Statistical Signal Processing: Estimation Theory, Englewood Cliffs, NJ: Prentice-Hall, 1993.

[12] Y.R. Zheng, C. Xiao, Simulation models with correct statistical properties for Rayleigh fading channels, Communications, 2003, 51(6): 920-928.

[13] M. Ghogho, S. Ananthram, Improved channel estimation using superimposed training, In: Proc. of IEEE 5th Workshop on Signal Processing Advances in Wireless Communications, Jul. 11-14, 2004: 110-114.

[14] X.D. Cai, G.B. Giannakis, Bounding performance and suppressing intercarrier interference in wireless mobile OFDM, Communications, 2003. 51(12): 2047-2056.

[15] A. Stamoulis, S.N. Diggavi, N. Al-Dhahir, Intercarrier interference in MIMO OFDM, Signal Process, 2002, 50(10): 2451-2464.

[16] P. Schniter, Low-complexity equalization of OFDM in doubly-selective channels, Signal Process, 2004, 52(4): 1002-1011.

[17] A. Gorokhov, J.P. Linnartz, Robust OFDM receivers for dispersive time-varying channels: Equalization and channel acquisition, Communications, 2004, 52(4): 572-583.

(Editor: Dongju CHEN) 